\title{
Nanowires Network for Biomolecular Detection Using Contactless Impedance Tomoscopy Technique
}

\author{
J. Gamby, ${ }^{\dagger}$ J.-P. Abid, ${ }^{\dagger}$ M. Abid, ${ }^{\prime}$ J.-P. Ansermet, ${ }^{\ddagger}$ and H. H. Girault*,† \\ Laboratoire d'Electrochimie Physique et Analytique, ISIC-Station 6, and Laboratoire de Physique des Materiaux \\ Nanostructurés, IPN-Station 3, Ecole Polytechnique Féderale de Lausanne, CH-1015 Lausanne, Switzerland
}

\begin{abstract}
Here we present the detection of ultralow concentrations of biomolecules in a device made from a polycarbonate membrane containing a network of gold nanowires and using a "contactless" impedance tomoscopy technique. The sensor comprises a thin dielectric layer with two parallel band electrodes on the one side and a microchannel containing gold nanowires onto which the adsorption of antibodies occurs. Upon applying a highfrequency ac voltage between the two electrodes, the adsorption process occurring at the surface of the gold nanowires can be followed through contactless impedance measurements. The configuration allows the real-time detection of biomolecules with a bulk concentration in the picomolar range.
\end{abstract}

In the past decade, an increasing interest has been dedicated to the development of optical and electrical biosensors. ${ }^{1-5}$ Several configurations have been envisaged to design optimized sensors. ${ }^{6-10}$ One of them consists of using the particular electronic, optical, and catalytic properties of metallic, semiconducting nano-objects. In the field of medicine and biology, the unique properties of nanomaterials have opened new ways of developing new tools for probing ultralow concentrations of biomolecules, amino acids, or DNA. ${ }^{11-14}$ The most widely used optical technique is called surface

* Corresponding author. Tel.: +41.21.693.31.51. Fax: +41.21.693.36.67. E-mail: hubert.girault@epfl.ch.

$\dagger$ Laboratoire d'Electrochimie Physique et Analytique.

* Laboratoire de Physique des Matériaux Nanostructurés.

(1) Hahm, J.; Lieber, C. M. Nano Lett. 2004, 4 (1), 51-54.

(2) Cui, Y.; Wie, Q.; Paak, H.; Lieber, C. M. Science 2001, 293, 1289-1292.

(3) Song, X.; Swanson, B. I. Anal. Chem. 1999, 71 (11), 2097-2107.

(4) Haes, A. J.; Chang, L.; Klein, W. L.; Van Duyne, R. P. J. Am. Chem. Soc. 2005, 127 (7), 2264-2271.

(5) Ferguson, J. P.; Baxter, G. A.; McEvoy, J. D. G.; Stead, S.; Rawlings, E.; Sharman, M. Analyst 2002, 127 (7), 951-956.

(6) Hafeman, D. G.; Parce, J. W.; McConnell. H. M. Science 1988, 240 (4856), 1182-1185.

(7) Zhou, Y.-M.; Wu, Z.-Y.; Shen, G.-L.; Yu, R.-Q. Sens. Actuators, B 2003, 89 (3), 292-298.

(8) Gallardo, S. A. M.; Jaffari, S. A.; Bone, S. Biosens, Bioelectron. 2001, 16 (1-2), 23-29.

(9) Cooper, M. A. Nat. Rev. 2002, 1 (7), 515-528.

(10) Ziegler, K. J. Trends Biotechnol. 2005, 23 (9), 440-444.

(11) Niemeyer, C. M. Angew. Chem., Int. Ed. 2001, 40 (22), 4128-4158.

(12) Alivisatos, P. Nat. Biotechnol. 2004, 22 (1), 47-52.

(13) Wang, J. Small 2005, 1 (11), 1036-1043.

(14) Englehenne, P.; Van Hoonacker, A. Curr. Nanosci. 2005, 1 (2), 97-106.

10.1021/ac060479z CCC: $\$ 33.50$ C 2006 American Chemical Society Published on Web 06/30/2006 plasmon resonance. The latter is based on the measurement of the dielectric constant changes induced by molecular interactions at the surface of a noble metal film. ${ }^{15-18}$ For ultralow concentration, the small changes of the refractive index can limit the detection character of this technique. To improve the detection sensitivity, the use of materials with high and variable dielectric constants has been envisaged. ${ }^{19,20}$ In 2002, He and co-workers introduced the concept of using gold nanoparticles for the detection of DNA hybridization. ${ }^{21}$ The combination of nanotechnology with the surface plasmon resonance technique lowers the limit of detection in the picomolar range without any difficulty. Others optical techniques such as confocal fluorescence microscopy and nonlinear optical null ellipsometry have also been envisaged for designing ultrasensitive sensors. ${ }^{21,23}$

Recently, new devices using a nanowire-based field effect transistor (FET) have been designed for the detection of chemical and biological species. This system consists of planar semiconductors in a $\mathrm{p} / \mathrm{n} / \mathrm{p}$ configuration in contact with a membrane where the biomolecules are immobilized. This configuration can be easily electrically, optically, and physically monitored in order to modify the sensitivity of the device. ${ }^{24,25}$ For example, planar FETs can be configured as sensors by modifying the gate oxide with molecular receptors or a selective membrane for the analyte of interest. ${ }^{26}$ In that configuration, the adsorption of charged species at the surface of the gate oxide results in the accumulation of carriers within the transistor structures and hence modifies the electrical proper-

(15) Homola, J.; Yu, S. S.; Myszka, D. In Optical Biosensors: Present and Future; Liger, F. S., Rowe Taitt, C. A., Eds.; Elsevier: Amsterdam, 2002; pp 207251.

(16) Homola, J. Anal. Bioanal. Chem. 2003, 377, 528-539.

(17) Chen, S.; Liu, L.; Zhou, J.; Jiang, S. Langmuir 2003, 19 (7), 28592864.

(18) Rich, R. L.; Day, Y. S. N.; Morton, T. A.; Myszka, D. G. Anal. Biochem. 2001, 296 (2), 197-207.

(19) Zhu, X.-M.; Lin, P.-H.; Ao, P.; Sorensen, L. B. Sens. Actuators, B 2002, 84 (2-3), 106-112.

(20) Chien, F.-C.; Chen, S.-J. Biosens. Bioelectron. 2004, 20 (3), 633-642.

(21) Nie, L.; Chen, H.; Tan, M.; He, N. J. Southeast Univ. 2002, 20 (4), 463466.

(22) Gruner, G. Anal. Bioanal. Chem. 2006, 384 (2), 322-335.

(23) Ruckstuhl, T.; Walser, A.; Verdes, D.; Seeger, S. Biosens. Bioelectron. 2005, 20 (9), 1872-1877.

(24) Star, A.; Gabriel, J.-C. P.; Bradley, K.; Gruener, G. Nano Lett. 2003, 3 (4), 459-463.

(25) Galla, H. J. Angew. Chem. 1992, 104 (1), 47-49.

(26) Assadi, A.; Willander, M.; Svensson, C.; Hellberg, S. Synth. Met. 1993, 58 (2), 187-193 
ties or the conductance of the system. This information can be easily followed and is dependent to the total concentration of charged species. ${ }^{27}$ Although the good sensitivity of the FET approach, the time-consuming fabrication and the limitation to one species for each device with a specific membrane limit the performance of the system.

In the present approach, a gold nanowire network embedded in a membrane is employed as a template for the detection of biomolecules using a noncontact impedance technique. The surface of the dielectric substrate in contact with the solution has been modified physically by techniques such as UV laser photoablation and plasma etching in order to generate a channel through the matrix. The detection concept is based on establishing a capacitive coupling between electrodes placed on one side of the dielectric substrate and the solution in contact with the other side. Compared with the FET system and the biodevices available, the surface specificity of the capacitive admittance tomoscopy allows the detection of biomolecules with concentration in the picomolar range and has several advantages: the configuration is low-cost; the microelectrodes are located outside the microchannel (noncontact mode) so that any undesired phenomena at the surface of the electrodes such as bubble generation or electrical passivation are avoided. The impedance tomoscopy is a surface-sensitive technique. Hence, the sensitivity is related to the surface area of the probed system. In that context, a large surfaceto-volume ratio in the channel can dramatically increase the sensitivity. Finally, the system may be employed for a "multiuse and multivariety" detection due to the fact that, in a low concentration regime, the adsorption of biomolecules on modified gold nanomaterials is reversible and depends on the electrostatic state of the microchannel surface. ${ }^{28}$ Since most of the biological molecules, such as proteins and DNA, are charged under physiological conditions, the device is appropriate to detect and study the adsorption of a variety of biomolecules onto the surface of the nanowires.

\section{MATERIALS AND METHODS}

The formation of the gold nanowires into the channel has been achieved using a $20-\mu \mathrm{m}$-thick, nuclear track etched polycarbonate membranes with a pore diameter of $50 \mathrm{~nm}$. A gold layer was deposited into the pores by using a gold cyanide salt solution. Prior to use, the membrane was rinsed under ultrasonic agitation. This step is crucial to ensure the wetting of the pores and the homogeneity of the growth rate over the working electrode area. ${ }^{29-31}$ The electrochemical experiments were performed using an EG\&G Princeton Applied Research model 263 potentiostat/ galvanostat in a standard type three-electrode electrolytic configuration. A platinum wire of $2.5 \mathrm{~mm}$ in diameter was used as counter electrode and a classical silver-silver chloride $(\mathrm{Ag} / \mathrm{AgCl})$ electrode was employed as reference. The working electrode consists of the sputtered gold layer of 500-nm thickness on one

(27) Bergveld, P. Biosensors 1986, 2 (1), 11-33.

(28) Gamby, J.; Abid, J. P.; Girault, H. H. J. Am. Chem. Soc. 2005, 127, 1330013304.

(29) Valizadeh, S.; Leisner, P.; Hultman, S.; George J. M. Adv. Funct. Mater. 2002, 12 (11-12), 766-772.

(30) Valizadeh, S.; George, J. M.; Leisner, P.; Hultman, L. Thin Solid Films 2002, 402, 262-271.

(31) Valizadeh, S.; George, J. M.; Leisner, P.; Hultman, L. Electrochim. Acta 2001, 47, 6 .

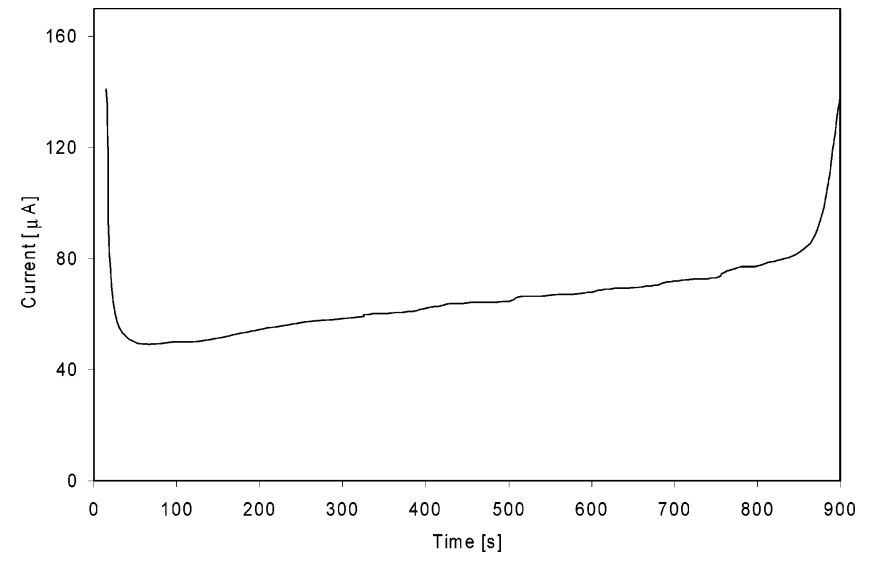

Figure 1. $1-t$ curve transient during synthesis of $A u$ nanowires at $E_{\mathrm{Au}}=-690 \mathrm{mV}$ versus $\mathrm{Ag} / \mathrm{AgCl}$.

side of the membrane. The electrodeposition process of $\mathrm{Au}$ nanowires is achieved at $E_{\mathrm{Au}}=-690 \mathrm{mV}$ in a standard gold solution containing $0.8 \mathrm{M}$ ascorbic acid and $0.3 \times 10^{-3} \mathrm{M} \mathrm{Au}$ $\left(\mathrm{KAu}(\mathrm{CN})_{2}\right)$ and at $\mathrm{pH} 3.5-4$. Using chronoamperometry, the $\mathrm{Au}$ nanowire growth process is followed until complete pore filling. The current response during reduction of $\mathrm{Au}^{+}$ions at constant applied potential, $E_{\mathrm{Au}}=-690 \mathrm{mV}$, is displayed in Figure 1 . The reduction process is completed when the current rapidly increases.

In a preliminary stage, a decrease of the current density is recorded during the process of the gold deposition, which is related to the rate of electrochemical processes, mainly the effect of mass transport by diffusion-controlled limiting current. Instantaneously reduced electroactive species establish a continuous depleted layer in the vicinity of the electrode. Accordingly, a concentration gradient is formed in the electrolyte between the nanoelectrodes ${ }^{31}$ and the bulk concentration. At longer times, the Au metal is growing inside the pores and the distance to the pore opening becomes smaller, which gives rise, consequently, to a slightly increasing current density. As a result, gold ions move rapidly toward the electrode surface by the radial diffusion from the bulk solution to the partially depleted diffusion zone. Finally, when the pores are filled up on the top of membrane surface, a cap formation associated with three-dimensional deposition arises. The hemispherical caps originating from each nanowire form a coherent planar layer that expanded to cover the whole surface of the membrane. Consequently, the effective cathode area increases and a rapidly increasing deposition current can be observed. For the present application, the electrodeposition process is stopped before reaching this stage.

The morphology and size distribution of the nanowires have been studied using a transmission electron microscope (TEM; Philips CM20). The TEM sample is prepared by dissolving the membrane in dichloromethane and collecting the wires on a TEM copper grid. TEM measurements show a narrow size distribution of gold nanowires with a diameter of $50 \mathrm{~nm}$ (SD, $4.0 \mathrm{~nm})$. The corresponding TEM micrograph is displayed in Figure 2. The inset in Figure 2 shows an electron diffraction pattern taken from one of the Au grains. The wires have even edges and exhibit a bamboo structure with grains elongated in the growth direction of the nanowires. XRD analysis of $20-\mu \mathrm{m}$-thick polycarbonate membranes electrodeposited with Au nanowire arrays is portrayed in Figure 3. XRD analysis of a virgin membrane with a 500-nm sputter- 


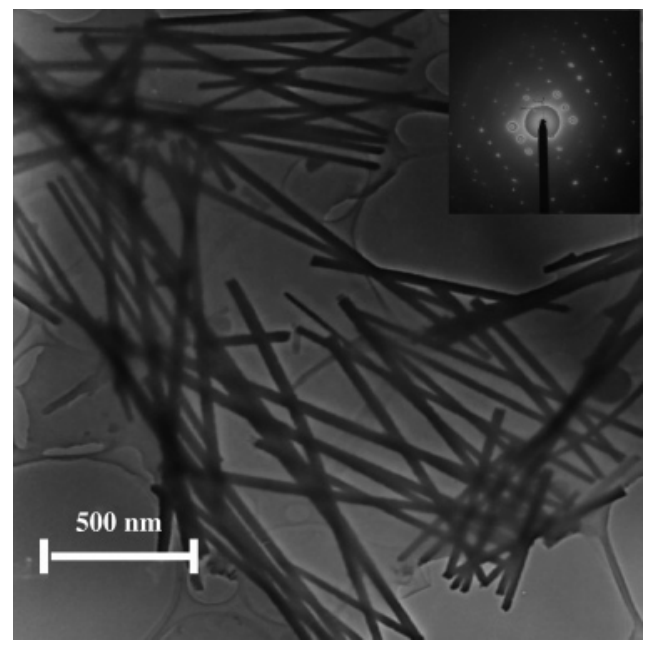

Figure 2. TEM micrograph of gold nanowires prepared using the electrodeposition technique with the corresponding diffraction pattern of a gold nanowire (inset).

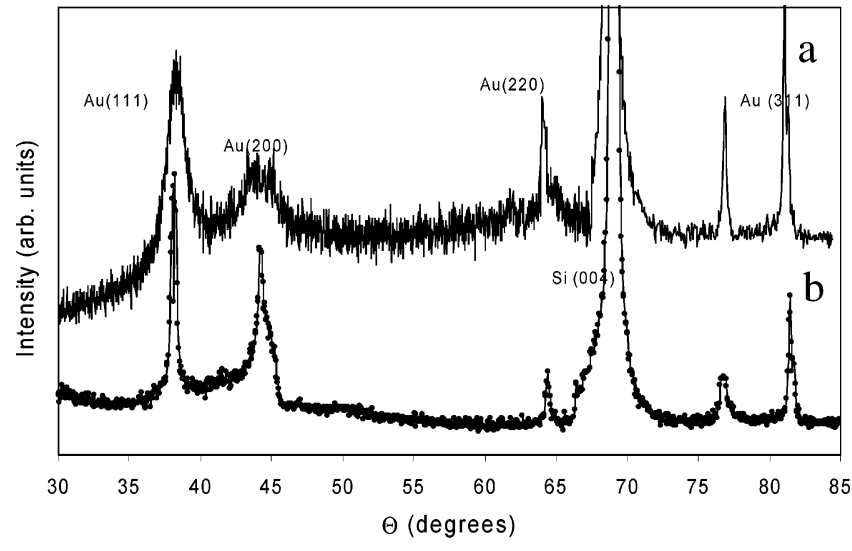

-Electrodeposited Au Nanowires $\rightarrow$ Sputter deposited membrane with Au

Figure 3. $\theta-2 \theta$ XRD patterns of (a) electrochemically deposited Au nanowires in ion track etched polycarbonate membrane and (b) a polycrystalline sputter deposited on Au layer with fcc structure. The strong peak from $\mathrm{Si}(004)$ is related to the substrate holder.

deposited Au layer is used as a comparison layer. The sputtered Au layer exhibits a polycrystalline fcc structure with preferential $\langle 111\rangle$ crystallite orientation. By comparison with the reference membrane, the polycrystalline fcc (111), (200), and (220) peaks are from the electrodeposited Au electrode.

On this filled template, two layers of polyethylene-terephthalate (35 $\mu \mathrm{m}$ thick, Morane, Oxon, UK) were thermally laminated on both sides at $135{ }^{\circ} \mathrm{C}$ and at a pressure of 2 bar (see PET1 and PET2 in Figure 5). The PET/PC gold nanowire/PET system is exposed to an $\mathrm{ArF}$ excimer laser beam in order to define a 35- $\mu$ m-deep cavity, which reaches the surface of the polycarbonate. To remove the polycarbonate membrane, an argon plasma etching with Ar pressure of 380 mbar and a power of $100 \mathrm{~W}$ is performed for $4 \mathrm{~h}$. By this process $15 \mu \mathrm{m}$ of polycarbonate was removed. SEM micrographs showing the nanowire network inside the microchannel is diplayed in Figure 4.

The channel structure is then sealed with a PET/polyethylene (PET/PE) lamination (see PET3 in Figure 5). The dimensions of the flow channel are $35 \times 100 \mu \mathrm{m}$ in cross section and a length of $1.5 \mathrm{~cm}$. On the other side of the film, two parallel microchannels with a depth of $30 \mu \mathrm{m}$ and a width of $100 \mu \mathrm{m}$ were photoablated perpendicularly to it as portrayed in Figure 5. The distance separation between these two parallel microchannels is $100 \mu \mathrm{m}$. The two channels were then filled with a mixture of commercial graphite ink and gold nanoparticles (Electra $\Omega$ ED5000 series obtained from Electra Polymers). After curing at $60^{\circ} \mathrm{C}$ for $4 \mathrm{~h}$, the device consists on a polycarbonate membrane with a groove of exposed nanowires sandwiched between two PET films (PET1 and PET3 in Figure 5), one of which holds two microelectrodes.

The real-time detection of a flowing solution is performed using a high-frequency admittance tomoscopy technique and applying an ac modulated signal with a frequency ranging from $1 \mathrm{kHz}$ to $1 \mathrm{MHz}$ and amplitude of $3 \mathrm{~V}$. A frequency response analyzer (1255B) combined with a dielectric interface 1296 (Solartron) that extends the frequency range from $10 \mu \mathrm{Hz}$ to $10 \mathrm{MHz}$. The current measurement range of the system varies from $6 \mathrm{~mA}$ to 100 $\mathrm{fA}$, thus enabling the measurement of the response of systems with high impedances $(100 \Omega-100 \mathrm{~T} \Omega)$ and low capacitances $(1 \mathrm{pF}$ to higher than $0.1 \mathrm{~F}$ ). All the acquisition is made using the company made software SMaRT. In that configuration, the measured current can thus be related to the total admittance of the biosensor.

\section{RESULTS AND DISCUSSION}

To study the sensitivity of the bioelectrochemical sensor, a set of experiments with variable concentrations of IgG immunoglobulins have been performed. All the experiments are made without any further modification of the gold nanowires. To ensure the reversibility of the adsorption, the study has been limited to IgG concentration lower than $1 \mu \mathrm{M}$. The evolution of the impedance with respect to the $\mathrm{IgG}$ concentration was measured at $1 \mathrm{MHz}$. (Figure 6). Increasing the concentration of IgG from $6.6 \times 10^{-15}$ to $6.6 \times 10^{-7} \mathrm{M}$ induces the decrease of the impedance until a plateau is reached for IgG immunoglobulin concentrations higher than $6.6 \times 10^{-7} \mathrm{M}$. The evolution of the impedance is consistent with the charging process of the metal nanowires by the adsorption of charged immnoglobulins. That is, the surface coverage with charged IgG increases until a full monolayer is reached. At a frequency of $1 \mathrm{MHz}$, a limit of detection of $6.6 \times$ $10^{-15} \mathrm{M}$ with an excellent reproducibility can be easily achieved $(0.089 \%$ o0 $)$. In comparison with available amperometric, ${ }^{2,32-35}$ optical biosensors, ${ }^{36-38}$ a lower limit of detection and better sensitivity are obtained. As an example, Cui et al. reported a nanosensor for the detection of biological and chemical species and based on the study of the conductance of semiconductor nanowires. Studying the adsorption of streptavidin, they reported a limit of detection in the picomolar range. ${ }^{2}$ Supercapacitive admittance tomoscopy allows the detection of impedance variation

(32) Gwan, J.-G.; Miao, Y.-Q.; Chen, J.-R. Biosens. Bioelectron. 2004, 19 (8), 789-794.

(33) Sarkar, P.; Pal, P. S.; Ghosh, D.; Stetford, S. J.; Johill, I. E. Int. J. Pharm. 2002, $238(1-2), 1-9$

(34) Dutra, R. F.; Coelho, G. D.; Silva, V. L.; Ledingham, W. M.; Limra, F. J. L. Biotechnol. Lett. 2000, 22 (7), 579-583.

(35) Dancil, K. P. S.; Grenier, D. P.; Sailor, M. J. J. Am. Chem. Soc. 1999, 121 (34), 7925-7930.

(36) Cunningham, B.; Lin, B.; Qiu, J.; Li, P.; Pepper, J.; Hugh, B. Sens. Actuators, B 2002, 85 (3), 219-226.

(37) Pieper-Furst, U.; Kleuser, U.; Stocklein, W. F. M.; Warsinke, A.; Scheller, F. W. Anal. Biochem. 2004, 332 (1), 160-167.

(38) Frau, P. T.; Parige, M. F. Bioanal. Chem. 2004, 380 (2), 339-342. 

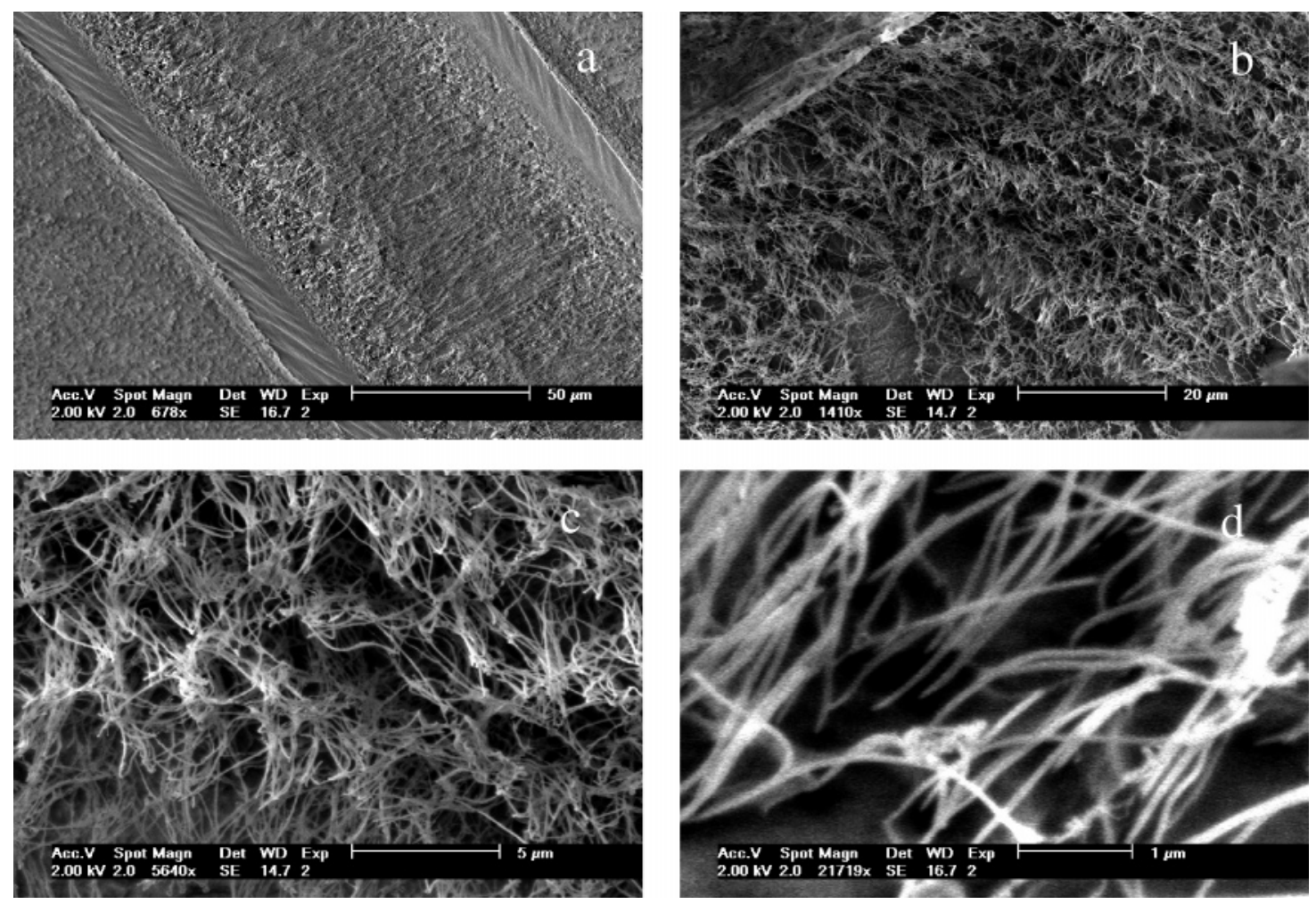

Figure 4. SEM micrographs of the microchannel at increasing magnification. (a) SEM micrograph at a magnification of 678. (b) SEM micrograph (1410× magnification). (c) At a magnification of 5640. (d) SEM micrograph (21719× magnification).

a

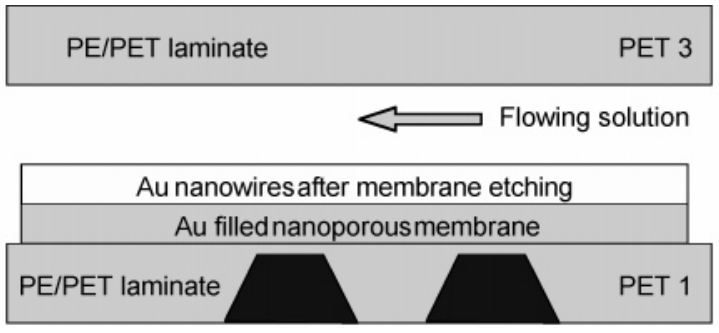

Figure 5. (a) Side view of the device. (b) Front view of the device.

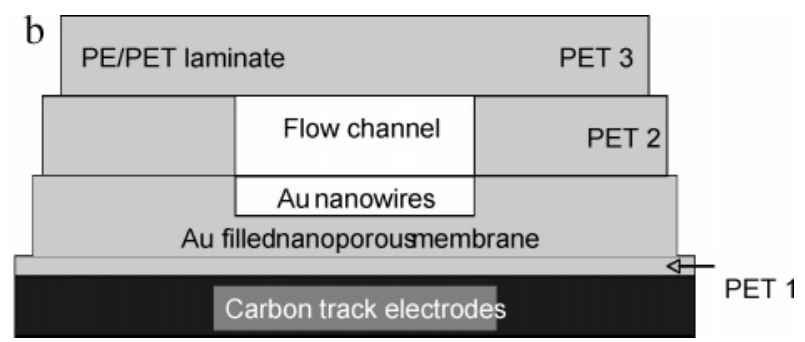

of $\sim 30 \Omega$ corresponding to the possible detection of samples with distinctive concentration in the order of $10^{-1}{ }^{4} \mathrm{M}$. Another important fact can be gleaned from Figure 6; the real-time monitoring of the impedance follows a Langmuir isotherm with a dissociation constant of $\sim 6.3 \times 10^{7} \mathrm{M}^{-1}$. This value is in good agreement with previous constants obtained for the study of antibodies onto the surface of metals. This shows clearly that the quantification of antibodies and the study of interactions of antibodies with the metals can be monitored in real time by this method.

The main process governing the electrical properties of the biodevice is the adsorption of charged IgG at the surface of the gold nanowire network. In fact, at the $\mathrm{pH}$ condition 7 , the $\operatorname{IgG}$ immunoglobulin ( $\mathrm{pI}=6.2)^{39}$ is negatively charged and its adsorption on the surface of the gold nanowires causes a change of the charged region in the vicinity of the metal. The behavior of our impedimetric system can be understood by analogy with an equivalent electrical circuit combining capacitors and resistors. In our approach, the system is divided into three domains: the "bulk" solution, which is characterized by a constant resistance
$R_{\mathrm{S}}=2220 \Omega$ and the interfacial region governed by the charging process of the nanowire surface through the adsorption of charged immunoglobulins. This process is introduced using a combination of a resistor $\mathrm{R}_{1}$ and a capacitor $\mathrm{C}_{1}$ in parallel. The capacitance $C_{1}$ is directly related to the permittivity of the capacitor: $C_{1}=$ $\tilde{\epsilon}(A / d)$, where $\tilde{\epsilon}$ is the effective dielectric function, $A$ is the area, and $d$ is the separation distance. Now, any adsorption of charged species will modify the effective dielectric function and thus the capacitance of the network. Finally, a supplementary contribution arises from the capacitive coupling between the electrode bands separated by a distance of $100 \mu \mathrm{m}$. This contribution has been introduced by considering a combination of a resistor $\mathrm{R}_{2}$ and a capacitor $\mathrm{C}_{2}$. This latter contribution is independent of the process occurring at the interface of the nanowires network and can be considered negligible. A schematic representation of the equivalent circuit envisaged for modeling the biodevice is shown i Chart 1.

(39) Davalos, L.; Ortersa-Vinuesa, J. L.; Bastos-Gonzalez, D.; Hidalgo-Alvarez R. J. Biomater. Sci., Polym. Ed. 2000, 11 (6), 657-673. 


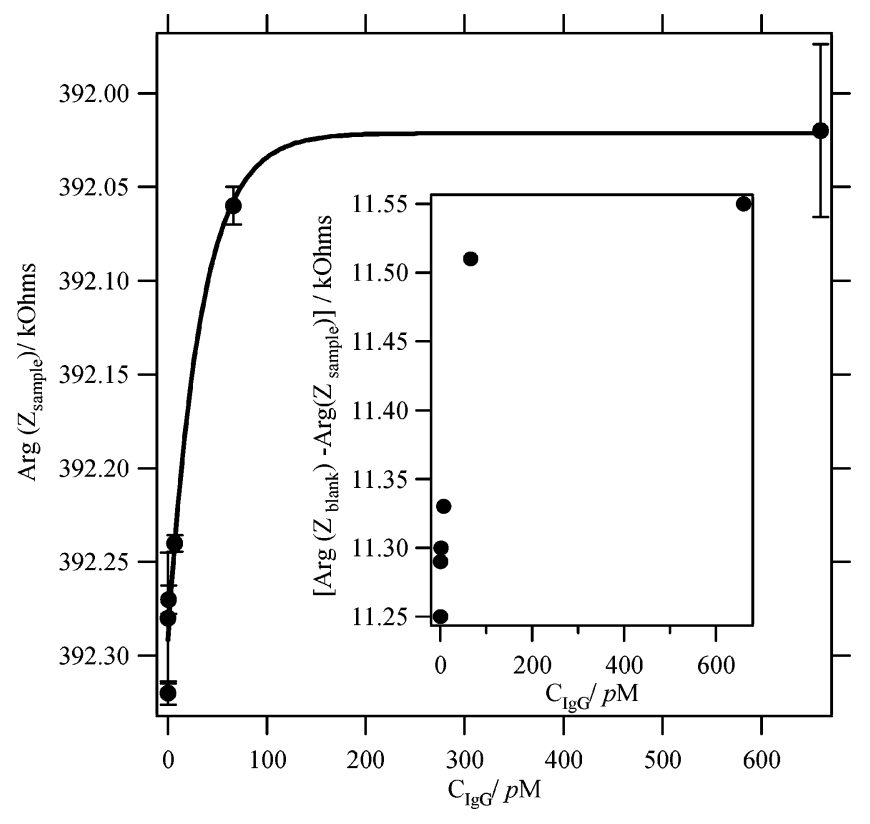

Figure 6. Evolution of the impedance modulus with respect to the bulk concentration at $1 \mathrm{MHz}$. Inset: corrected impedance modulus versus the IgG bulk concentration at $1 \mathrm{MHz}$. The correction is made by subtracting the impedance of the device filled with a buffer solution $\left(Z_{\text {blank }}=403.570 \mathrm{k} \Omega\right)$.

\section{Chart 1. Equivalent Circuit of the Biodevice}

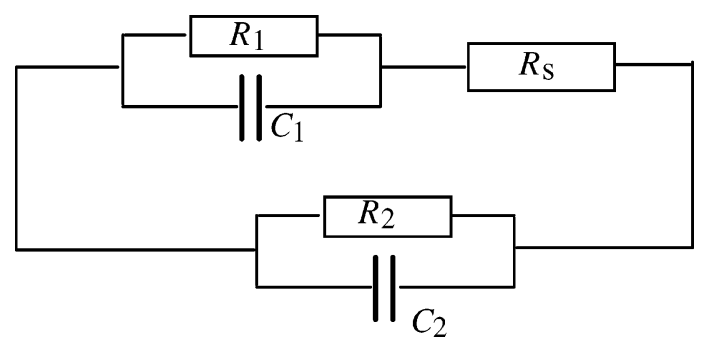

Considering the equivalent circuit, the total impedance can be derived:

$$
Z(\omega)=\frac{R_{2}\left(R_{\mathrm{S}}+R_{1}+j \omega R_{\mathrm{S}} R_{1} C_{1}\right)}{\left(1+j \omega R_{2} C_{2}\right)\left(R_{\mathrm{S}}+j \omega R_{\mathrm{S}} R_{1} C_{1}\right)+R_{2}\left(1+j \omega R_{\mathrm{S}} R_{1} C_{1}\right)}
$$

To study the response of the biodevice with respect to the angular frequency, the impedance modulus noted $\operatorname{Arg}(Z)$ is estimated. The experimental results are compared to the present model. For different concentrations of immunoglobulins, the evolution of the impedance with respect to the angular frequency is portrayed in Figure 7. Preliminary comparison is made with the impedimetric response of the device for IgG concentration of $6.6 \times 10^{-15} \mathrm{M}$ (Figure 7a). Using the equivalent circuit detailed previously, a good approximation has been reached for the following parameters: resistance of the solution $R_{\mathrm{S}}=2220 \Omega$, resistance of the interface $R_{1}=2 \times 10^{8} \Omega$, capacitance of the electrical interface layers $C_{1}=3.28 \times 10^{-13} \mathrm{~F}$, resistance of the PET material between the two microelectrodes $R_{2}=1 \times 10^{14} \Omega$, and the stray capacitance between the two microelectrodes $C_{2}=2 \times 10^{-16} \mathrm{~F}$. As expected, a high resistance and a low capacitance are obtained. Similar
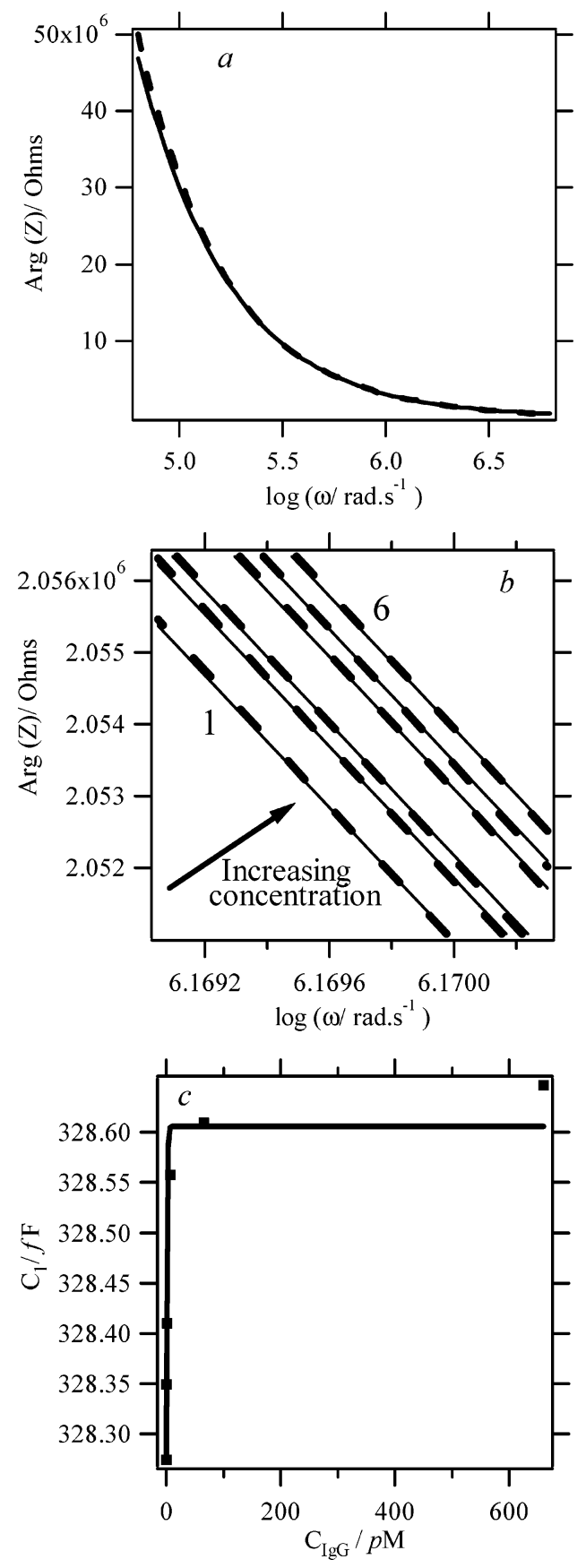

Figure 7. Static mode impedance measurement during the process of adsorption of the antibodies. (a) Experimental angular frequency response of the impedance (dotted line) and theoretical fit using eq 1 (solid line) yielding the following parameters: $R_{\mathrm{S}}=2220 \Omega, R_{1}=$ $2 \times 10^{8} \Omega, R_{2}=1 \times 10^{14} \Omega, C_{1}=3.28 \times 10^{-13} \mathrm{~F}$, and $C_{2}=2 \times$ $10^{-16} \mathrm{~F}$. (b) Simulation of the impedance of the device with different bulk concentrations of antibodies in order to obtain capacitance values at the interface. Dotted line from curves 1-6: $C_{\mathrm{lgG}}=6.6 \times 10^{-15}$, $6.6 \times 10^{-14}, 6.6 \times 10^{-13}, 6.6 \times 10^{-12}, 6.6 \times 10^{-11}$, and $6.6 \times 10^{-10}$ $\mathrm{M}$; solid line, best simulation of $C_{1}$ capacitance, in the case of the adsorption of antibodies onto the nanowires surface. Solid line from curves 1-6: $328.34 \times 10^{-15}, 328.27 \times 10^{-15}, 328.41 \times 10^{-15}, 328.56$ $\times 10^{-15}, 328.61 \times 10^{-15}$, and $328.74 \times 10^{-15} \mathrm{~F}$. (c) Experimental evolution of the capacitance at $1 \mathrm{MHz}$ with respect to the bulk concentration.

treatment is made on the impedance response of samples with the following concentrations: $6.6 \times 10^{-15}, 6.6 \times 10^{-14}, 6.6 \times 10^{-13}$, $6.6 \times 10^{-12}, 6.6 \times 10^{-11}$, and $6.6 \times 10^{-10} \mathrm{M}$. For each concentration, 


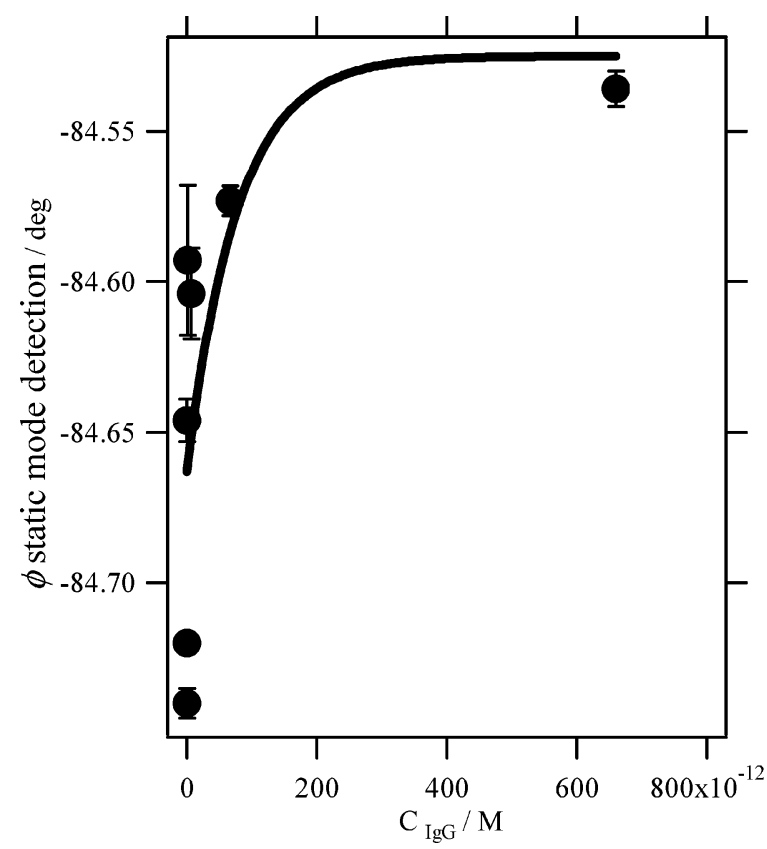

Figure 8. Impedance phase in a static mode detection of the adsorption of the antibodies measured after $8 \mathrm{~min}$, from bottom to the top: $C_{\mathrm{lgG}}=6.6 \times 10^{-16}, 6.6 \times 10^{-15}, 6.6 \times 10^{-14}, 6.6 \times 10^{-13}$, $6.6 \times 10^{-12}, 6.6 \times 10^{-11}$, and $6.6 \times 10^{-10} \mathrm{M}$.

a variation of the impedance is observed as portrayed in Figure 7b. The evolution of the capacitance with respect to the concentration of immunoglobulins is summarized in Figure 7c. An increase of the immunoglobulin concentration shows a noticeable increase of the capacitance of the biodevice. Typically, increasing the concentration of IgG immunoglobulins from $6.6 \times 10^{-15}$ to $6.6 \times$ $10^{-10} \mathrm{M}$ results in the increase of the capacitance from $328.34 \times$ $10^{-15}$ to $328.74 \times 10^{-15} \mathrm{~F}$. It must be pointed out that a $0.1 \%$ variation of capacitance can readily be detected by the impedance analyzer at $1 \mathrm{MHz}$.

The capacitance evolution is governed by the interface state and the processes involved at the interface. In the present approach, the interface is described using a capacitor with a variable capacitance $C_{1}$. This latter is dependent on the nature of the species adsorbed onto the surface of the nanowires. The unique electrical property of the device opens a new way for the detection of antibodies adsorbed onto the surface of gold nanowires. It must be pointed out that the capacitor character of the biodevice is confirmed by measuring the phase angle of the biodevice. The phase angle measurements are made on samples with concentration ranging from $C_{\mathrm{IgG}}=6.6 \times 10^{-16}$ to $6.6 \times 10^{-10}$ M (Figure 8). The biodevice presents a phase angle of about $84-85^{\circ}$, which is near the typical value of the capacitor. An increase of the IgG immunoglobulin concentration shows a slight decrease of the absolute value of the phase angle. The experimental phase angle can be compared to the phase angle of the equivalent circuit introduced in Chart 1.

The phase angle is estimated as

$$
\tan \phi=\operatorname{Im}(Z(\omega)) / \operatorname{Re}(Z(\omega))
$$

The experimental value of $-85^{\circ}$ is in good agreement with the theoretical value of $-90^{\circ}$ obtained using the equivalent circuit

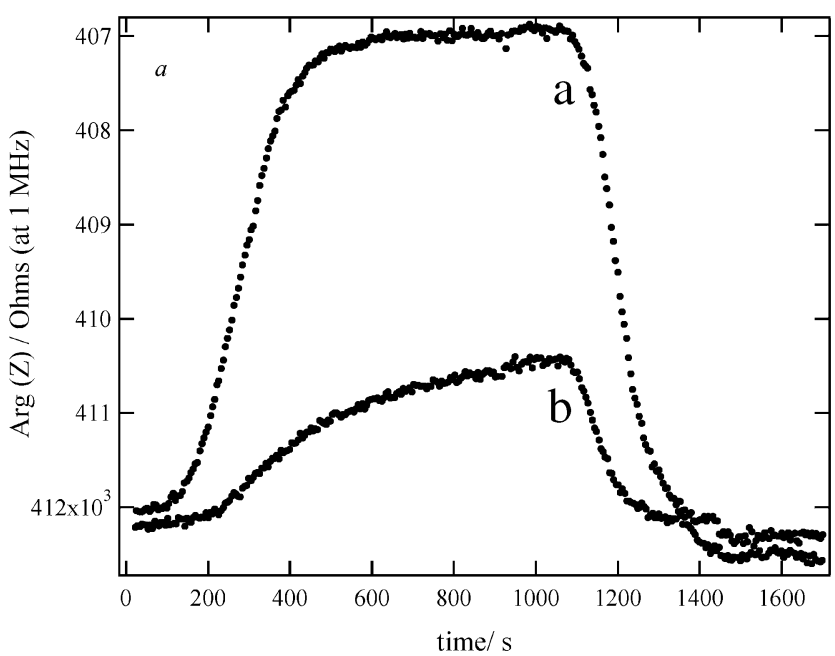

Figure 9. Sensorgrams of the adsorption of the antibodies. Impedance amplitude sensorgrams showing the real-time adsorption/ desorption of antibodies. Before each experiment, a preliminary step consists of filling the microchannel with PBS buffer to reach the baseline. Then in a second step, a sample of antibody is injected in a dynamic regime during $900 \mathrm{~s}$ for adsorption: (a) evolution of the impedance modulus for a sample with a concentration of $\mathrm{lgG}$ of 1.65 $\times 10^{-6} \mathrm{M}$; (b) evolution of the impedance modulus for a sample with a concentration of $6.6 \times 10^{-12} \mathrm{M}$. The desorption process is achieved by filling the channel with a buffer solution. For all injections, a typical flow of $0.1 \mu \mathrm{L} \cdot \mathrm{min}^{-1}$ is used. The fluidic is controlled by a dual syringe pump and a manual valve.

displayed in Chart 1 . The biodevice behaves like a tunable capacitor dependent on the nature and the concentration of the adsorbed species onto the surface of the nanowires. The design of the device allows the monitoring of the IgG adsorption at the surface of the gold nanowires in a real-time mode. An example of impedance sensorgrams for two concentrations is displayed in Figure 9. As previously reported, a decrease of the impedance is observed that corresponds to the process of the IgG adsorption at the surface of gold nanowires. After reaching equilibrium, the microchannel is washed with a buffer solution. The impedance decreases until it returns to its original value. This corresponds to the possible release of the IgG from the gold surface. The process of the reversibility of the $\operatorname{IgG}$ adsorption has been observed only for concentrations lower than $1 \mu \mathrm{M} .^{28}$

\section{CONCLUSIONS}

A biodevice combining a network of nanowires inside a microchannel and a high-frequency noncontact detection technique has been developed to detect ultralow concentration of biomolecules. In the case of IgG immunoglobulins, a bulk concentration of $\sim 6 \mathrm{fM}$ can be reached. The results show that the biodevice acts similarly to a capacitor with variable capacitance. The principle of capacitive admittance tomoscopy is based on the study of the capacitive coupling across the thin layer separating the band electrodes from the solution. By flowing a solution of charged species, the adsorption of the charged species (biomolecules) on the surface of the modified microchannel induces the modification of the capacitance of the device, and hence depending on the amplitude of the capacitance variation, the concentration of biomolecules can be determined. This coupling effect across the thin dielectric layer separating the band electrodes from the 
flowing solution can be related to a capacitor where the charges on one side are electronic charges and localized on the band electrodes and the charges on the solution side are ionic charges, either from the ionized groups generated on the polycarbonate supporting the nanowires or more generally by the adsorbed layer and the space charge region in solution, e.g., the Gouy-Chapman layer. Compared to classical impedimetric sensors using a metallic electrode in direct contact with the analyte, the originality of the present work comes from the fact that the electrode bands are in "noncontact" configuration, separated from the solution by a thin layer of PET with a thickness of $5 \mu \mathrm{m}$, but more important that the surface of the dielectric in contact with the solution has been decorated by an array of insulated gold nanowire to increase the specific surface area.

\section{ACKNOWLEDGMENT}

The authors thank Solartron Switzerland for the loan of the dielectric interface 1296.

Received for review March 16, 2006. Accepted June 13, 2006.

AC060479Z 